\title{
The Female Autism Phenotype and Camouflaging: a Narrative Review
}

\author{
Laura Hull ${ }^{1,2}$ (1) K. V. Petrides ${ }^{3} \cdot$ William Mandy $^{1}$
}

Received: 4 June 2019 / Accepted: 22 January 2020 / Published online: 29 January 2020

(C) The Author(s) 2020

\begin{abstract}
Autism is more commonly diagnosed in males than females. One explanation is the 'female protective effect': there is something inherent in being female which reduces the likelihood of developing autism. However, evidence suggests that the condition is underdiagnosed in females, perhaps because females express their autism in ways which do not meet current diagnostic criteria. This review explores evidence for a female-typical autism presentation, the Female Autism Phenotype (FAP) and the component of camouflaging (compensating for and masking autistic characteristics) in particular. The evidence so far supports the existence of a female-typical autism presentation, although further examination of the characteristics and their impact across all genders and ages is needed.
\end{abstract}

Keywords Autism $\cdot$ Gender $\cdot$ Sex $\cdot$ Camouflaging $\cdot$ Female protective effect $\cdot$ Female autism phenotype

\section{Autism Diagnosis and Prevalence}

Autism diagnosis has traditionally been most common in childhood, when differences from neurotypical peers may first become obvious. However, in recent years, there has been a significant increase in the rate of adult diagnosis, particularly as diagnostic criteria have been broadened such that individuals who may not have received an autism diagnosis in childhood may now meet current diagnostic criteria (Happé et al. 2016). Note: we use 'autism' to refer to the clinical diagnosis of autism spectrum disorder, as some members of the autism community feel the label 'disorder' produces stigma and emphasises the difficulties associated with autism while minimising the strengths. For similar reasons, we use identity-first language ('autistic person') throughout to respect the preferences of a majority of autistic people (Kenny et al. 2016). Changes to diagnostic criteria which are linked to an

Laura Hull

laura.hull.14@ucl.ac.uk

1 Research Department of Clinical, Educational \& Health Psychology, University College London, London, UK

2 Department of Psychology, University College London, 26 Bedford Way, London WC1H 0AP, UK

3 London Psychometric Laboratory, University College London, London, UK increase in overall diagnostic levels include the integration of previously separate diagnostic categories (such as 'Autism' and 'Asperger Syndrome') into one 'autism spectrum disorder' category (Murphy et al. 2016). There have been many studies examining the needs and experiences of individuals who seek an autism diagnosis in adulthood (Crane et al. 2018). In particular, there has been a focus on the difficulties experienced by autistic women in obtaining autism diagnoses, for reasons which will be discussed in greater detail below.

As there are no reliable biomarkers of autism, the condition is diagnosed behaviourally, based on observation and description of the core characteristics impacting everyday functioning to a 'clinically significant' degree (American Psychiatric Association 2013; World Health Organization 2018). Estimates of autism prevalence are continually updated. The most recent estimates suggest a prevalence of 1 in 69 children in America (Christensen et al. 2018), while in the UK, estimates are higher at 1 in 59 (Russell et al. 2014). Prevalence estimates are most often determined in western, higherdeveloped nations (e.g. Randall et al. 2016), and these tend to be higher than those estimated in low-income countries (Elsabbagh et al. 2012). This could suggest that there are significant numbers of autistic people in other nations, including African and Asian nations, who are not recognised by or receiving support from medical or educational systems (Hossain et al. 2017; Mpaka et al. 2016); alternatively, there may be lifestyle factors in more developed nations which lead to greater incidence of autism. 


\section{Gender Differences in Diagnosis}

Autism is more commonly diagnosed in males than in females across age groups (Fombonne 2009; Russell et al. 2011). When screening the entire population using gold standard assessments, current estimates suggest around three males receive an autism diagnosis for every female; however, in clinical samples who have already received an autism diagnosis, that ratio is higher at over four males to each female (Loomes et al. 2017). In individuals with intellectual disability, the ratio is closer to 2:1 (Yeargin-Allsopp et al. 2003).

When attempting to account for the discrepancies in diagnosis, researchers have drawn upon two distinct ideas, which are contrasting but not mutually exclusive. One argues that there is something inherent in being female that 'protects' females from the likelihood of developing autism (Robinson et al. 2013). The other proposes that females may be more likely to develop autism than we currently estimate, but that diagnostic biases and variation in the ways autism is expressed in females mean we do not pick up autism in females to the same degree as males (Dworzynski et al. 2012; Russell et al. 2011).

\section{Female Protective Effect}

The female protective effect theory (FPE theory) comes from research into proposed genetic and environmental factors affecting autism development. It proposes that females require greater environmental and/or genetic risk than males to express the same degree of autistic characteristics, and, hence, that females are 'protected' from autistic characteristics relative to males with a comparable level of risk factors (Robinson et al. 2013). In support of FPE, autistic females possess relatively more spontaneous, non-inherited mutations associated with autism than males (Gilman et al. 2011; Levy et al. 2011). Males and females in these studies had comparable levels of autistic characteristics, suggesting that a greater genetic hit is required for females to meet the diagnostic threshold. This implies an innate protective factor in females, which results in reduced behavioural expression of autistic characteristics when the genetic risk of autism is equivalent to that of males.

If, as FPE suggests, females are, on average, protected against autism compared to males, then autistic females should have greater genetic load than males in order to express the same level of characteristics. As the majority of variance in autism is inherited for both males and females (Tick et al. 2016), their close genetic relatives should also carry more genetic load for autism than the close relatives of autistic males; in other words, relatives of autistic females should be more likely to have autism, or autistic characteristics, than relatives of autistic males. Evidence for this hypothesis is mixed. While some studies suggest that autistic females' first degree relatives have more autistic characteristics than first-degree relatives of autistic males (Desachy et al. 2015; Frazier et al. 2015), others have found the opposite effect (Ozonoff et al. 2011).

A further limitation to FPE is that no specific protective factor has been conclusively demonstrated as yet. The extreme male brain theory (EMB theory) proposes that androgens and related sex hormones more common in males may underlie many autistic characteristics (BaronCohen 2002). Characteristics associated with autism, such as high levels of systemising abilities and difficulties with cognitive empathy and emotional expression tasks, are proposed to represent masculine characteristics, such that autistic individuals are presented as having 'extreme male' behavioural and psychological presentations (Baron-Cohen 2002). The EMB theory suggests that individuals with lower levels of androgens (i.e. females) also demonstrate lower levels of these characteristics, and therefore that having low levels of androgens is protective against autistic characteristics (Baron-Cohen et al. 2011, 2015). A relationship between autistic characteristics and high androgen levels in females has been found (Knickmeyer et al. 2006; Schwarz et al. 2011); however, other research suggests that foetal and early developmental androgen levels have a very limited relationship with autism diagnosis (Guyatt et al. 2015).

Another proposed source of the protective factor is the $\mathrm{X}$ chromosome, with a protective gene expressed on the paternal $\mathrm{X}$ chromosome for females, which increases the threshold for autism expression relatives to males (Skuse 2000); however, no specific protective gene has been identified here. Environmental factors such as in vitro exposure to medications seem to increase autism likelihood in males more than females (Harrington et al. 2014). These factors may interact with genetic risks to further increase autism likelihood in males, and hence increase relative autism protection in females. Further research is undoubtedly needed to identify the protective and risk factors involved in male and female autism development.

A key limitation of research into relative male and female risks of autism is that most studies assume current estimates of male and female diagnostic rates are accurate. As will be discussed in detail below, this may not necessarily be the case. If autistic females are in fact less likely to be diagnosed than are autistic males, despite demonstrating equivalent levels of autistic characteristics, there may be genetic or behavioural differences between those females who do receive an autism diagnosis and those who do not. Evidence from the FPE comes from studies of autistic females who meet current diagnostic criteria, using current diagnostic tools. Females who do not meet current criteria using these tools, for reasons that will be discussed below, may also have lower genetic risk, or display genetic variations that have not yet been associated 
with autism. Conclusions as to the generalisability of the FPE to all autistic females must, therefore, be limited until it can be demonstrated that a truly representative sample of autistic females are included in these genetic analyses.

\section{Female Autism Phenotype}

Even if biological factors exist to reduce the likelihood of autism in females, there is also substantial evidence suggesting that diagnostic processes are less likely to identify females, particularly those without intellectual disability (Russell et al. 2011). Females require more additional difficulties than males to receive an autism diagnosis, despite having equivalent levels of autistic characteristics (Duvekot et al. 2017; Dworzynski et al. 2012; Shattuck et al. 2009). Clinical samples appear to underestimate the number of females, as male/ female ratios are significantly higher in these than in population-based samples in which all individuals are screened for autism (Loomes et al. 2017). In addition, females who do receive an autism diagnosis do so at a later age than males on average (Begeer et al. 2013; Kirkovski et al. 2013; Rutherford et al. 2016). A fundamental issue with the current diagnostic procedure is that behavioural markers used as diagnostic criteria are established based on pre-existing conceptions of what autistic behaviours look like. These criteria have been developed based on the predominantly male populations previously identified as autistic (Kirkovski et al. 2013; Kopp and Gillberg 2011; Mattila et al. 2011). Females may be less likely to meet these criteria even when clinically significant characteristics are identified, resulting in diagnoses of broader developmental disorders, rather than autism specifically (Wilson et al. 2016).

Individual characteristics may interact with gender to further reduce the likelihood of females receiving an autism diagnosis. Females with low IQ are more likely to receive a diagnosis than females with high IQ (Rivet and Matson 2011; Van Wijngaarden-Cremers et al. 2014). Total rates of autism diagnosis have increased over time, representing a broadening of diagnostic criteria to include individuals with greater variation in presentation (Brugha et al. 2011; Saemundsen et al. 2013), although increased diagnoses appear to be mostly those of boys without intellectual disability (Blumberg et al. 2013). There is also some evidence to suggest that adult women are seeking and receiving autism diagnoses to a greater extent than men (Happé et al. 2016), supporting the argument that these women were even more likely to be missed at a younger age than their male peers.

One explanation for the underdiagnosis of females is that their presentation of autism is qualitatively different to the typical male presentation. This female autism phenotype (FAP), or behavioural expression of autism more common in females, represents similar underlying autistic characteristics as those described in current diagnostic criteria (i.e. difficulties with social communication and interaction, restricted interests and repetitive behaviours and unusual sensory responses). However, these characteristics may be expressed in ways that differ from traditional autism diagnostic criteria (Hull et al. 2017b; Kirkovski et al. 2013; Lai et al. 2011). Females may also express other, additional behaviours or characteristics that are not currently included in autism diagnostic criteria. Some of these behaviours and characteristics have been previously assessed with regard to the female autism phenotype (Hull et al. 2017b); those with the greatest evidence are briefly discussed here.

\section{Social Relationships}

Difficulties with social relationships, particularly friendships, are a hallmark of autism (American Psychiatric Association 2013); the FAP theory suggests that the nature of these difficulties may differ depending on an individual's gender. Some research has suggested that autistic females may have fewer social impairments than males; autistic females tend to have higher levels of social motivation (the desire and intent to form friendships with others) than males on average (Head et al. 2014; Hiller et al. 2014). However, autistic females may find it harder to maintain long-term friendships or relationships than autistic males, despite having similar levels of motivation for social relationships as non-autistic females (Hiller et al. 2014). Conflict in social relationships may also be harder for autistic females to cope with than autistic males or non-autistic females (Sedgewick et al. 2019). These findings suggest that in addition to assessing the maintenance of social relationships, research into female autistic experience should also compare to non-autistic female characteristics to obtain an accurate evaluation of relative social abilities. When compared to autistic males, females may appear to have fewer social difficulties overall, but may struggle with other aspects of socialising, especially in comparison to non-autistic females.

\section{Relational Interests}

In a similar vein, some research into gender differences in restricted and repetitive interests has suggested that autistic females have lower levels of these interests than males (Hattier et al. 2011; Lai et al. 2015). However, some have argued that autistic females' special interests may be in different areas to males, and so may be underestimated, if these areas are not probed during assessments or are not considered 'atypical' (Antezana et al. 2019; Mandy et al. 2012). Further research explicitly comparing the nature of males' and 
females' special interests in autism appears to support this. Studies have found that autistic males' interests tend to be focused on more mechanical topics such as vehicles, computers or physics (Grove et al. 2018; Nowell et al. 2019). On the other hand, autistic females' interests appear to focus more on topics with relational purposes, such as animals, fictional characters or psychology (Grove et al. 2018; Mandy et al. 2012; McFayden et al. 2018; Nowell et al. 2019).

While the intensity of the interest itself may be atypical for both genders, the type of interest may be considered more age and gender appropriate for females than males, and so may not be reported as unusual by parents, teachers or clinicians (Lai et al. 2015; Sutherland et al. 2017). Alternatively, because the interest is seen as more appropriate, it may create fewer difficulties for the autistic individual and their family, and not be considered clinically significant. Nevertheless, the differential nature of special interests may result in underestimation of autistic characteristics for autistic females, and so reduce the likelihood of a clinical diagnosis.

\section{Internalising Problems}

The previous two characteristics of the female autism phenotype concern differential expression of the core underlying features of autism. The next characteristic, namely internalising problems, concerns features which may form part of the typical clinical presentation, but do not represent the core features of autism. In contrast to externalising problems (where difficulties are turned outwards, resulting in aggression and difficulties relating with others), internalising problems describe the inward expression of emotional difficulties, including anxiety, depression, self-harming and eating disorders (Kovacs and Devlin 1998; Leadbeater et al. 1999). Most research demonstrates that autistic females are significantly more likely to have co-occurring internalising disorders than males, and these increase in severity at a greater extent than for males, although some studies with younger children find no variation between genders (Chandler et al. 2016; Gotham et al. 2015; Mandy et al. 2012; Oswald et al. 2016). Autistic males are significantly more likely to have cooccurring externalising disorders such as behavioural problems and inattention (Hiller et al. 2014; May et al. 2012).

There are two ways in which this may impact the identification of female autism. Firstly, more severe expression of these co-occurring conditions may serve to mask underlying autistic characteristics, such that females receive a diagnosis of the co-occurring condition only and their autism goes unrecognised. This has been reported anecdotally by many autistic women who received an autism diagnosis later in adulthood, after earlier diagnoses of internalising conditions (Bargiela et al. 2016). Secondly, if males tend to express their co-occurring conditions in more disruptive ways than females, this may perpetuate the gender bias in autism recognition and referral, particularly in school. The needs of autistic males may come to teachers' attention sooner and be seen as more intrusive, than females, who may be seen as shy or simply anxious (Hiller et al. 2014). Females' internalising difficulties may, therefore, not only leave them more vulnerable to serious mental health conditions, but also reduce the likelihood of their autism being recognised.

\section{Camouflaging}

One aspect of the female phenotype which has, until recently, received relatively limited attention, is the phenomenon of camouflaging. Camouflaging refers to the use of conscious or unconscious strategies, which may be explicitly learned or implicitly developed, to minimise the appearance of autistic characteristics during a social setting (Hull et al. 2017a; Lai et al. 2011). Examples include mimicking facial expressions of the person you are talking to (whether consciously or not), or forcing oneself to make eye contact and to stop talking about an interest. A similar concept which has been recently proposed is that of compensation (Livingston and Happé 2017). Compensation describes the use of alternative cognitive strategies to overcome specific socio-cognitive or behavioural difficulties in autism. For instance, an autistic individual might compensate for theory of mind difficulties by using executive function strategies to learn to recognise different facial expressions. Compensation can be shallow (involving external changes without affecting the underlying cognitive processes) or deep (involving alternative cognitive routes to achieve the desired outcome; Livingston and Happé 2017), and evidence of varying levels of compensation has been presented in autistic individuals (Livingston et al. 2019). In this paper, we include compensation strategies within broader camouflaging, while acknowledging that not all camouflaging involves alternative cognitive strategies.

Some of the first references to autistic camouflaging or to similar concepts appear in sources attempting to describe or explain the gender disparity in diagnosis, especially amongst individuals without intellectual impairment. As early as 1981, Lorna Wing hypothesised that some autistic girls with no intellectual disability may be missed in clinical assessments and that this may be related to females appearing to have better social and communication abilities compared to males (Wing 1981). This has come to be known as the 'camouflaging hypothesis'. She also described case studies of male and female individuals using strategies to learn rules or social behaviours, for example from television shows or books, which may appear typical at first glance and may make diagnosis harder. Historically, autistic women writing about their own experiences have also described behaviours and outcomes conceptually related to camouflaging; Liane Holliday Wiley 
described "pretending to be normal" (Holliday Willey 2015) for many years before receiving her diagnosis. However, these accounts were not always considered by clinicians or academics seeking to understand the female autistic experience.

The hypothesis of specific strategies being used by girls to mask autistic social difficulties was also noted in case studies by Kopp and Gillberg (1992). Again, the emphasis was on superficially typical social behaviour, but identification of underlying difficulties upon further examination. The authors suggested that these behaviours represented part of a 'female phenotype' of Aspergers, which might require adapted assessment tools or thresholds. This concept has been extended in later work seeking to identify more subtle presentations of social and communication difficulties in autistic girls (Kopp and Gillberg 2011).

Descriptions of camouflaging at this stage of conceptualisation focused on personal and clinical anecdotes, or hypotheses produced in the discussion section of related studies. Some specific behaviours that may represent camouflaging were proposed, although up to 2015, these ideas had not been tested in large-scale, high-quality studies. Suppressing physical characteristics such as hand flapping, giving scripted responses to questions and imitating others' facial expressions were all proposed as possible methods of camouflaging based on case studies and clinician or researcher experience (Kreiser and White 2014; Lai and Baron-Cohen 2015; Mandy and Tchanturia 2015). Strategies, such as staying close to other girls to avoid standing out (Gould and Ashton-Smith 2011), or adapting to school environments so difficulties were not identified by teachers (Hiller et al. 2014; Mandy et al. 2012) have also been proposed.

Although camouflaging had not yet been directly measured at that time, some suggestions were formed as to the mechanisms involved. It was proposed that autistic girls and women might identify and learn appropriate behaviours from others, especially peers, and that social expectations and reinforcements might have a greater influence on females than males in many cultures, increasing the importance of displaying the 'right' behaviours (Kreiser and White 2014; Lai et al. 2015, 2011). In addition, some suggested that genetic 'protective' effects might give females greater abilities to compensate for their autistic difficulties, potentially to the extent of not requiring an autism diagnosis (Dworzynski et al. 2012; Kirkovski et al. 2013).

The concept of varying adaptation to different environments - or variation in the person-environment fithas been suggested as a fundamental drive of camouflaging behaviours (Lai and Baron-Cohen 2015; Lai et al. 2015). Individuals who do not feel as though they fit into a social situation, particularly females who feel greater pressure to fit in socially, may attempt to adapt their behaviour in that situation in order to better fit in. In less-pressured situations, the same individual may not feel any need to adapt or compensate for their behaviours. This may lead to variation in presentation across different environments, for example at home, school or in clinical assessment, leading to a discrepancy in the perceived extent of autistic social communication difficulties. This may also account for variation in perceived autistic characteristics over time; the complexity of social situations may change across development, such that an autistic individual is able to adapt to or compensate for difficulties in more simple social interactions, but their difficulties may become more apparent as the social environment becomes more complex (Lai et al. 2011; Mandy et al. 2018).

\section{Research into Camouflaging}

Summarising the state of research into autism sex/gender differences in 2015, Lai et al. (2015) suggested that future research required the thorough operationalisation of camouflaging as well as the development of camouflaging assessment tools. Since then, several researchers have used qualitative methods to explore the concept of camouflaging, and multiple approaches to measuring camouflaging have been developed. These will now be briefly discussed.

A number of studies have used qualitative methods to investigate social camouflaging behaviours in autistic girls and women. Interviews with mothers of autistic girls (Cridland et al. 2014) described how some girls mimic their peers' social behaviours and interests, and it was suggested that this may reduce the appearance of autistic characteristics. Tierney et al. (2016) interviewed 10 adolescent autistic girls about their experiences of using 'coping strategies' and revealed some common themes including the uncertain, exhausting nature of the social environment; the desire to make friends which motivated camouflaging attempts; and using explicit techniques to mask autism-related difficulties. Similar themes have also been noted in qualitative interviews with late-diagnosed autistic women (Bargiela et al. 2016), and with autistic young women and their parents (Milner et al. 2019; Sedgewick et al. 2019; Sutherland et al. 2017). In particular, the idea of pretending to be normal, which could be achieved through both learned and automatic strategies, and the extensive costs of such strategies, were identified.

A conceptual model of camouflaging was developed through open-response questions with a large sample of autistic adults (Hull et al. 2017a). Synthesising the responses of 92 autistic adults of all genders, clear themes regarding the motivation for and consequences of camouflaging were identified, along with themes summarising types of camouflaging behaviours. Motivations for camouflaging in this study comprised the desire to fit in with others, to avoid bullying or other negative treatment; and wanting to form connections with others which the autistic person felt was not possible when they presented as their authentic self. Camouflaging 
behaviours involved masking of autistic characteristics by presenting more socially acceptable personas; and compensating for differences in social presentation, such as forcing oneself to make eye contact. Consequences of camouflaging included physical and emotional exhaustion, often requiring time alone to recover; issues around identity and authenticity which led some participants to "lose track of who I really am" (Hull et al. 2017a; p 2530); and difficulty accessing support and diagnosis. These findings suggested that experiences of camouflaging are heterogeneous but common across the autistic community, and stimulated the development of quantitative measures of camouflaging to allow comparison between different individuals.

\section{Discrepancy Approach}

Attempts to measure camouflaging have mostly fallen under two different approaches (see Table 1 for a summary of the approaches and studies following them). The first focuses on camouflaging as evidenced by the discrepancy between an individual's innate autistic characteristics and their external presentation of autism.

Some researchers have conceptualised camouflaging as the difference between the core difficulties or characteristics associated with autism, and the behavioural presentation of autism in a specific situation (Lai et al. 2017; Livingston and Happé 2017). This could be described as a 'discrepancy' approach to camouflaging, as it seeks to operationalise the discrepancy between how autistic a person truly is (their internal autistic status), and how autistic they appear to be externally (their external autistic presentation).

Empirical studies using this discrepancy approach (summarised in Table 1) have generally found that autistic females have higher camouflaging discrepancy scores than males. For instance, females were found to have a greater discrepancy between self-reported autistic traits and social cognitive abilities, and autistic behaviours as measured by an observer (Lai et al. 2017). This discrepancy was also associated with greater activation of the ventromedial prefrontal cortex in response to self-representation in females only (Lai et al. 2019). A similar result was found by Ratto

Table 1 Summary of studies into camouflaging

\begin{tabular}{|c|c|c|}
\hline Approach & \multicolumn{2}{|l|}{ Description } \\
\hline \multirow[t]{8}{*}{ Discrepancy } & \multicolumn{2}{|c|}{ Conceptualises camouflaging as the discrepancy between an individual's internal and external autistic characteristics } \\
\hline & Studies using this approach & Operationalisation of camouflaging \\
\hline & Lai et al. 2017 & $\begin{array}{l}\text { Discrepancy between self-reported autistic traits/performance on mentalising task } \\
\text { (internal) and ADOS score (external) }\end{array}$ \\
\hline & Lai et al. 2019 & $\begin{array}{l}\text { Discrepancy between self-reported autistic traits/performance on mentalising } \\
\text { task (internal) and ADOS score (external) }\end{array}$ \\
\hline & Livingston et al. 2019 & $\begin{array}{l}\text { Discrepancy between performance on a theory of mind task (internal) and } \\
\text { ADOS score (external) }\end{array}$ \\
\hline & Parish-Morris et al. 2017 & $\begin{array}{l}\text { Discrepancy between parent-reported autistic traits (internal) and use of typical } \\
\text { language techniques (external) }\end{array}$ \\
\hline & Ratto et al. 2018 & $\begin{array}{l}\text { Discrepancy between parent-reported autistic traits/adaptive behaviour (internal), } \\
\text { and ADOS or ADOS-2 and ADI-R score (external) }\end{array}$ \\
\hline & Rynkiewicz et al. 2016 & $\begin{array}{l}\text { Discrepancy between self/parent-reported autistic traits (internal) and use of gesture } \\
\text { in ADOS-2 (external) }\end{array}$ \\
\hline \multirow[t]{10}{*}{ Observational/ Reflective } & \multicolumn{2}{|c|}{$\begin{array}{l}\text { Conceptualises camouflaging as the specific behaviours and processes (whether conscious or implicit) leading } \\
\text { to variation in the behavioural presentation of autism }\end{array}$} \\
\hline & Studies using this approach & Operationalisation of camouflaging \\
\hline & Cage et al. 2018 & Self-reported camouflaging (yes/no) \\
\hline & Cage and Troxell-Whitman 2019 & Score on self-report measure of camouflaging behaviours (CAT-Q). \\
\hline & Cassidy et al. 2018 & Score on four self-report questions of camouflaging \\
\hline & Dean et al. 2017 & Observed social behaviours in the playground \\
\hline & Hull et al. 2017a & Self-reported behavioural examples \\
\hline & Hull et al. 2018 & Score on self-report measure of camouflaging behaviours (CAT-Q). \\
\hline & Hull et al. 2019 & Score on self-report measure of camouflaging behaviours (CAT-Q). \\
\hline & Ormond et al. 2018 & $\begin{array}{l}\text { Score on 'social masking' subscale of parent-report autism characteristic } \\
\text { questionnaire (Q-ASC). }\end{array}$ \\
\hline
\end{tabular}


et al. (2018), where autistic females had higher levels of parent-reported autistic traits and poorer adaptive functioning than males, despite being matched on scores of observer-rated autistic characteristics.

Other discrepancy approaches have looked at specific characteristics of autistic behavioural expression. For instance, autistic females were found to use more typical patterns of filling pauses in conversation than males of equivalent autistic trait severity (Parish-Morris et al. 2017). Another study found trends towards autistic girls using more vivid gestures in computerised ADOS- 2 than boys, where both genders reported equivalent levels of autistic traits (Rynkiewicz et al. 2016).

These approaches are strengthened by the way in which they operationalise camouflaging; the extent of camouflaging (as measured by numerical discrepancy) can be compared between groups and across studies using different techniques and measuring different behaviours. However, a limitation of the discrepancy approach is that measures of 'internal' autistic characteristics are at best proxies for 'true' internal characteristics. There currently exist no reliable biomarkers for autism (American Psychiatric Association 2013; Loth et al. 2015), and currently used self-report and informant-report measures of autistic characteristics are themselves subject to potential biases or misinterpretation (Kopp and Gillberg 2011; Kreiser and White 2014).

Discrepancy approaches seek to measure the impact of camouflaging on external presentation of autism; however, this approach does not account for the impact of unsuccessful camouflaging attempts. Camouflaging strategies, whether conscious or unconscious, may have varying success for different individuals across different situations, and attempts may not be as successful as the individual would wish. Preliminary research into camouflaging suggests that it is associated with poor mental health outcomes, including anxiety, depression, and suicidal thoughts (Cage et al. 2018; Cage and TroxellWhitman 2019; Cassidy et al. 2018; Hull et al. 2018; Lai et al. 2017). It therefore seems necessary to measure both camouflaging intention and success, and to be able to separate the two, to understand the full impact of camouflaging across an autistic individual's lifespan.

\section{Observational/Reflective Approach}

An alternative approach to measure camouflaging focuses on the direct identification of camouflaging behaviours through observation and reflection by autistic individuals or others around them. Studies using this approach are summarised in Table 1. Extent of camouflaging can therefore be quantified without the need for a measure of internal autistic characteristics, as it is the behaviours themselves rather than the underlying characteristics they might mask which are of interest. Similarly to discrepancy approaches, this allows for comparison across individuals (assuming they use the same methods of observation or reporting) and has the additional strength of allowing measurement of behaviours regardless of how successful they are. In other words, identification of camouflaging is not reliant on either a proxy measure of internal autistic status, or the need to display a typical social presentation.

Several reliable and psychometrically valid measures of camouflaging behaviours have been developed recently. One of these is the Camouflaging Autistic Traits Questionnaire (CAT-Q; Hull et al. 2018), designed to measuring camouflaging behaviours in adults. This measure has demonstrated equivalence of structure across autistic and non-autistic men and women, which allows for comparison across diagnostic groups and genders. The CAT-Q represents another advantage of observational/reflective approaches to measuring camouflaging; it is grounded in a conceptualisation of camouflaging based on autistic individuals' own experiences (Hull et al. 2017a). Another is the social masking subscale of the Questionnaire for Autism Spectrum Conditions (Q-ASC; Ormond et al. 2018), a parent-report measure which has been validated in children without intellectual disability. As a result, these measures of camouflaging have greater relevance to autistic individuals' actual experience of camouflaging, and therefore can be applied in clinical and individual settings to help autistic people understand more about their own camouflaging behaviours.

With regards to gender differences in camouflaging, studies using the observational/reflection approach have found inconsistent results. Camouflaging behaviours were observed in autistic girls in the playground, but not autistic boys or typically developing children of either gender (Dean et al. 2017). Parent-reported measures reveal that autistic girls use more social masking and imitation strategies than boys (Ormond et al. 2018). When asked to report whether or not they camouflage, no gender differences have been observed in autistic adults (Cage et al. 2018; Cassidy et al. 2018; Hull et al. 2017a). One study using the CAT-Q has found no difference in total score between males and females (Cage and TroxellWhitman 2019), while another study found autistic adult women scored higher than men, with no significant difference between non-binary individuals and males or females (Hull et al. 2019). Other self-report measures of camouflaging suggest females have higher total scores (Cassidy et al. 2018). More research is needed using a variety of observational/ reflective measures to determine whether camouflaging is more common in autistic females than males, and therefore whether it forms part of the female autism phenotype.

\section{Future Directions for Research}

Research into camouflaging using either discrepancy or observational/reflective approaches is still very much in its infancy. However, there are some particular areas of 
future research which should be addressed. Firstly, much research into camouflaging in autism has focused on the experiences of autistic adults, or of younger children based on parent-reported behaviours. Camouflaging behaviours are likely to develop across the lifespan, but may have significant impact during later childhood and adolescence as individuals learn patterns of behaviour used for the rest of their lives. Research should aim to identify when and how camouflaging behaviours first develop, particularly when considering the potential negative outcomes over the longer term.

Secondly, most research into gender and camouflaging has used a binary approach to gender, comparing the experiences and behaviours of males and females. While this is of relevance to the concept of a female autism phenotype, in contrast to the often male-biased diagnostic criteria and assessment tools, this approach ignores the substantial minority of autistic individuals who report a non-binary or fluid gender identity (Cooper et al. 2018; Dewinter et al. 2017). It also raises issues regarding the appropriateness of male and female norms for transgender autistic individuals, who may have experiences of living and interacting as different genders in different stages of life (Strang et al. 2018).

Finally, future research into camouflaging should acknowledge that motivations and expectations to camouflage may vary across different situations or stages of a person's life. Some camouflaging strategies may be adaptive and produce positive outcomes for the individual such as helping them to connect with others or to overcome specific cognitive difficulties associated with their autism (Livingston and Happé 2017). Other strategies, or the same strategies used under different circumstances, may lead to negative outcomes or perpetuate an expectation of adaptation to others' expectations which reduces autistic individuals' self-esteem (Hull et al. 2017a). Rather than categorising camouflaging as either a positive or negative behaviour within the female autism phenotype (and for non-female autistic individuals), researchers and clinicians whose aim is to improve autistic people's lives should approach camouflaging at an individual level, working with each person to identify which aspects of their behaviours are helpful and which are harmful.

\section{Conclusions}

Discrepancies in autism diagnosis between males and females may be due both to reduced likelihood of autism development in females, and under-diagnosis of those females who are autistic. One explanation for the latter is that some autistic females express their autism through subtle variations in behaviour compared to males, which are not captured in current diagnostic tools or criteria.
This review summarised some evidence for the proposed female autism phenotype, and focused on the concept of camouflaging as an important topic of research in recent years. The included evidence generally supports the existence of a female autism phenotype, which may include differences in social difficulties regarding the maintenance of social relationships; interests which tend to be more relational in nature; and the co-occurrence of internalising disorders. However, further research is necessary to determine whether other behaviours may also comprise the female autism phenotype and to explore whether these characteristics represent a uniquely female expression of autism, or simply variation from the stereotypical autistic behaviours identified in earlier years.

It is still unclear whether camouflaging forms part of the female autism phenotype. Future research into camouflaging should utilise reliable, accurate measures of both camouflaging intention and camouflaging success, which can be used with individuals of all genders, ages and abilities. This will enable further examination of potential gender differences in camouflaging, both in overall extent and in consequences, which should be extended beyond the binary gender divide and across ages. These attempts would benefit from the identification of neural or biological measures of innate autistic characteristics, in order to distinguish these from the external behavioural presentation of autism.

The findings summarised in this review suggest that, overall, camouflaging is a relatively common part of the everyday experiences of autistic individuals without intellectual disability. This has implications for clinical and research settings, particularly regarding the accurate diagnosis of autism and identification of required support. Autistic individuals may camouflage their autistic characteristics during clinical assessments, consciously or not, which may lead to missed diagnosis or lack of appropriate support. Clinicians and those involved in assessment of autistic characteristics should be made aware of the possibility of camouflaging, and should consider this particularly when assessing individuals who appear close to the threshold for a diagnosis of autism. Clinicians should also be aware of the strong associations between camouflaging and poor mental health outcomes, and take this into account when identifying support strategies for autistic individuals across the lifespan.

Acknowledgements This article was adapted from Laura Hull's doctoral dissertation at University College London.

\section{Compliance with Ethical Standards}

Conflict of Interest The authors declare that they have no conflict of interest.

This article does not contain any studies with human participants or animals performed by any of the authors. 
Open Access This article is licensed under a Creative Commons Attribution 4.0 International License, which permits use, sharing, adaptation, distribution and reproduction in any medium or format, as long as you give appropriate credit to the original author(s) and the source, provide a link to the Creative Commons licence, and indicate if changes were made. The images or other third party material in this article are included in the article's Creative Commons licence, unless indicated otherwise in a credit line to the material. If material is not included in the article's Creative Commons licence and your intended use is not permitted by statutory regulation or exceeds the permitted use, you will need to obtain permission directly from the copyright holder. To view a copy of this licence, visit http://creativecommons.org/licenses/by/4.0/.

\section{References}

American Psychiatric Association. (2013). DSM 5. American Psychiatric Association.

Antezana, L., Factor, R. S., Condy, E. E., Strege, M. V., Scarpa, A., \& Richey, J. A. (2019). Gender differences in restricted and repetitive behaviors and interests in youth with autism. Autism Research, 12(2), 274-283. https://doi.org/10.1002/aur.2049.

Bargiela, S., Steward, R., \& Mandy, W. (2016). The experiences of latediagnosed women with autism Spectrum conditions: An investigation of the female autism phenotype. Journal of Autism and Developmental Disorders, 46(10), 3281-3294. https://doi.org/10. 1007/s10803-016-2872-8.

Baron-Cohen, S. (2002). The extreme male brain theory of autism. Trends in Cognitive Sciences, 6(6), 248-254. https://doi.org/10.1016/ S1364-6613(02)01904-6.

Baron-Cohen, S., Lombardo, M. V., Auyeung, B., Ashwin, E., Chakrabarti, B., \& Knickmeyer, R. (2011). Why are autism spectrum conditions more prevalent in males? PLoS Biology, 9(6), e1001081. https://doi.org/10.1371/journal.pbio.1001081.

Baron-Cohen, S., Auyeung, B., Nørgaard-Pedersen, B., Hougaard, D. M., Abdallah, M. W., Melgaard, L., et al. (2015). Elevated fetal steroidogenic activity in autism. Molecular Psychiatry, 20(3), 369-376. https://doi.org/10.1038/mp.2014.48.

Begeer, S., Mandell, D., Wijnker-Holmes, B., Venderbosch, S., Rem, D., Stekelenburg, F., \& Koot, H. M. (2013). Sex differences in the timing of identification among children and adults with autism spectrum disorders. Journal of Autism and Developmental Disorders, 43, 1151-1156.

Blumberg, S. J., Bramlett, M. D., Kogan, M. D., Schieve, L. A., Jones, J. R., \& Lu, M. C. (2013). Changes in prevalence of parent-reported autism spectrum disorder in school-aged U. S. children: 2007 to 2011-2012. National Health Statistics Reports, (65), 1-11.

Brugha, T. S., McManus, S., Bankart, J., Scott, F., Purdon, S., Smith, J., et al. (2011). Epidemiology of autism Spectrum disorders in adults in the Community in England. Archives of General Psychiatry, 68(5), 459-466. https://doi.org/10.1001/archgenpsychiatry.2011.38.

Cage, E., \& Troxell-Whitman, Z. (2019). Understanding the reasons, contexts and costs of camouflaging for autistic adults. Journal of Autism and Developmental Disorders, 49(5), 1899-1911. https:// doi.org/10.1007/s10803-018-03878-x.

Cage, E., Di Monaco, J., \& Newell, V. (2018). Experiences of autism acceptance and mental health in autistic adults. Journal of Autism and Developmental Disorders, 48(2), 473-484. https://doi.org/10. 1007/s10803-017-3342-7.

Cassidy, S., Bradley, L., Shaw, R., \& Baron-Cohen, S. (2018). Risk markers for suicidality in autistic adults. Molecular Autism, 9(42), 1-14. https://doi.org/10.1186/s13229-018-0226-4.
Chandler, S., Howlin, P., Simonoff, E., O'Sullivan, T., Tseng, E., Kennedy, J., et al. (2016). Emotional and behavioural problems in young children with autism spectrum disorder. Developmental Medicine and Child Neurology, 58(2), 202-208. https://doi.org/10. 1111/dmen.12830.

Christensen, D. L., Braun, K., Baio, J., Bilder, D., Charles, J., Constantino, J. N., et al. (2018). Prevalence and characteristics of autism Spectrum disorder among children aged 8 years - Autism and developmental disabilities monitoring network, 11 sites, United States, 2012. MMWR. Surveillance Summaries : Morbidity and Mortality Weekly Report. Surveillance Summaries / CDC, 65(13), 1-23. https://doi.org/10.15585/mmwr.ss6513a1.

Cooper, K., Smith, L. G. E., \& Russell, A. J. (2018). Gender identity in autism : Sex differences in social affiliation with gender groups. Journal of Autism and Developmental Disorders, 48(12), 39954006. https://doi.org/10.1007/s10803-018-3590-1.

Crane, L., Batty, R., Adeyinka, H., Goddard, L., Henry, L. A., \& Hill, E. L. (2018). Autism Diagnosis in the United Kingdom : Perspectives of Autistic Adults , Parents and Professionals. Journal of Autism and Developmental Disorders, 48(11), 3761-3772. https://doi.org/10. 1007/s10803-018-3639-1.

Cridland, E. K., Jones, S. C., Caputi, P., \& Magee, C. A. (2014). Being a girl in a boys' world: Investigating the experiences of girls with autism spectrum disorders during adolescence. Journal of Autism and Developmental Disorders, 44(6), 1261-1274. https://doi.org/ 10.1007/s10803-013-1985-6.

Dean, M., Harwood, R., \& Kasari, C. (2017). The art of camouflage: Gender differences in the social behaviors of girls and boys with autism spectrum disorder. Autism, 21(6), 678-689. https://doi.org/ $10.1177 / 1362361316671845$.

Desachy, G., Croen, L. A., Torres, A. R., Kharrazi, M., Delorenze, G. N., Windham, G. C., et al. (2015). Increased female autosomal burden of rare copy number variants in human populations and in autism families. Molecular Psychiatry, 20(2), 170-175. https://doi.org/10. 1038/mp.2014.179.

Dewinter, J., de Graaf, H., \& Begeer, S. (2017). Sexual orientation, gender identity, and romantic relationships in adolescents and adults with autism Spectrum disorder. Journal of Autism and Developmental Disorders, 47(9), 1-8. https://doi.org/10.1007/ s10803-017-3199-9.

Duvekot, J., Van der Ende, J., Verhulst, F. C., Slappendel, G., Van Daalen, E., Maras, A., \& Greaves-Lord, K. (2017). Factors influencing the probability of a diagnosis of autism Spectrum disorder in girls versus boys. Autism, 21(6), 646-658. https://doi.org/10.1177/ 1362361316672178 .

Dworzynski, K., Ronald, A., Bolton, P., \& Happé, F. (2012). How different are girls and boys above and below the diagnostic threshold for autism spectrum disorders? Journal of the American Academy of Child and Adolescent Psychiatry, 51(8), 788-797. https://doi.org/ 10.1016/j.jaac.2012.05.018

Elsabbagh, M., Divan, G., Koh, Y. J., Kim, Y. S., Kauchali, S., Marcín, C., et al. (2012). Global prevalence of autism and other pervasive developmental disorders. Autism Research, 5(3), 160-179. https:// doi.org/10.1002/aur.239.

Fombonne, E. (2009). Epidemiology of pervasive developmental disorders. Pediatric Research, 65(6), 591-598. https://doi.org/10.1203/ PDR.0b013e31819e7203.

Frazier, T. W., Youngstrom, E. A., Hardan, A. Y., Georgiades, S., Constantino, J. N., \& Eng, C. (2015). Quantitative autism symptom patterns recapitulate differential mechanisms of genetic transmission in single and multiple incidence families. Molecular Autism, 6, 58. https://doi.org/10.1186/s13229-015-0050-z.

Gilman, S. R., Iossifov, I., Levy, D., Ronemus, M., Wigler, M., \& Vitkup, D. (2011). Rare De novo variants associated with autism implicate a large functional network of genes involved in formation and 
function of synapses. Neuron, 70(5), 898-907. https://doi.org/10. 1016/j.neuron.2011.05.021.

Gotham, K., Brunwasser, S. M., \& Lord, C. (2015). Depressive and anxiety symptom trajectories from school age through young adulthood in samples with autism spectrum disorder and developmental delay. Journal of the American Academy of Child and Adolescent Psychiatry, 54(5), 369-376.e3. https://doi.org/10. 1016/j.jaac.2015.02.005.

Gould, J., \& Ashton-Smith, J. (2011). Missed diagnosis or misdiagnosis? Girls and women on the autism spectrum. Good Autism Practice (GAP), 12(1), 34-41.

Grove, R., Hoekstra, R. A., Wierda, M., \& Begeer, S. (2018). Special interests and subjective wellbeing in autistic adults. Autism Research, 11(5), 766-775. https://doi.org/10.1002/aur.1931.

Guyatt, A. L., Heron, J., Knight, B. L. C., Golding, J., \& Rai, D. (2015). Digit ratio and autism spectrum disorders in the Avon longitudinal study of parents and children: A birth cohort study. BMJ Open, 5(8), e007433. https://doi.org/10.1136/bmjopen-2014-007433.

Happé, F. G., Mansour, H., Barrett, P., Brown, T., Abbott, P., \& Charlton, R. A. (2016). Demographic and cognitive profile of individuals seeking a diagnosis of autism Spectrum disorder in adulthood. Journal of Autism and Developmental Disorders, 46(11), 34693480. https://doi.org/10.1007/s10803-016-2886-2.

Harrington, R. A., Lee, L.-C., Crum, R. M., Zimmerman, A. W., \& HertzPicciotto, I. (2014). Prenatal SSRI use and offspring with autism Spectrum disorder or developmental delay. Pediatrics, 133(5), e1241-e1248. https://doi.org/10.1542/peds.2013-3406.

Hattier, M. A., Matson, J. L., Tureck, K., \& Horovitz, M. (2011). The effects of gender and age on repetitive and/or restricted behaviors and interests in adults with autism spectrum disorders and intellectual disability. Research in Developmental Disabilities, 32(6), 23462351. https://doi.org/10.1016/j.ridd.2011.07.028.

Head, A. M., McGillivray, J. A., \& Stokes, M. A. (2014). Gender differences in emotionality and sociability in children with autism spectrum disorders. Molecular Autism, 5(19). https://doi.org/10.1186/ 2040-2392-5-19.

Hiller, R. M., Young, R. L., \& Weber, N. (2014). Sex differences in autism spectrum disorder based on DSM-5 criteria: Evidence from clinician and teacher reporting. Journal of Abnormal Child Psychology, 42(8), 1381-1393. https://doi.org/10.1007/s10802014-9881-x.

Holliday Willey, L. (2015). Pretending to be Normal: Living with Asperger's syndrome (Expanded ed.). London: Jessica Kingsley Publishers.

Hossain, M. D., Ahmed, H. U., Jalal Uddin, M. M., Chowdhury, W. A., Iqbal, M. S., Kabir, R. I., et al. (2017). Autism Spectrum disorders (ASD) in South Asia: A systematic review. BMC Psychiatry, 17(1), 1-7. https://doi.org/10.1186/s12888-017-1440-x.

Hull, L., Petrides, K. V., Allison, C., Smith, P., Baron-Cohen, S., Lai, M., \& Mandy, W. (2017a). "Putting on my best normal": Social camouflaging in adults with autism Spectrum conditions. Journal of Autism and Developmental Disorders, 47(8), 2519-2534. https:// doi.org/10.1007/s10803-017-3166-5.

Hull, L., Mandy, W., \& Petrides, K. V. (2017b). Behavioural and cognitive sex/gender differences in autism spectrum condition and typically developing males and females. Autism, 21(6), 706-727. https:// doi.org/10.1177/1362361316669087.

Hull, L., Mandy, W., Lai, M. C., Baron-Cohen, S., Allison, C., Smith, P., \& Petrides, K. V. (2018). Development and validation of the camouflaging autistic traits questionnaire (CAT-Q). Journal of Autism and Developmental Disorders, 49(3), 819-833. https://doi. org/10.1007/s10803-018-3792-6.

Hull, L., Lai, M.-C., Baron-Cohen, S., Allison, C., Smith, P., Petrides, K. V., \& Mandy, W. (2019). Gender differences in self-reported camouflaging in autistic and non-autistic adults. Autism, 136236131986480. https://doi.org/10.1177/1362361319864804.
Kenny, L., Hattersley, C., Molins, B., Buckley, C., Povey, C., \& Pellicano, E. (2016). Which terms should be used to describe autism? Perspectives from the UK autism community. Autism, 20(4), 442-462. https://doi.org/10.1177/1362361315588200.

Kirkovski, M., Enticott, P. G., \& Fitzgerald, P. B. (2013). A review of the role of female gender in autism spectrum disorders. Journal of Autism and Developmental Disorders, 43(11), 2584-2603. https:// doi.org/10.1007/s10803-013-1811-1.

Knickmeyer, R., Baron-Cohen, S., Fane, B. A., Wheelwright, S., Mathews, G. A., Conway, G. S., et al. (2006). Androgens and autistic traits: A study of individuals with congenital adrenal hyperplasia. Hormones and Behavior, 50(1), 148-153. https://doi.org/10. 1016/j.yhbeh.2006.02.006.

Kopp, S., \& Gillberg, C. (1992). Girls with social deficits and learning problems: Autism, atypical Asperger syndrome or a variant of these conditions. European Child \& Adolescent Psychiatry, 1(2), 89-99. https://doi.org/10.1007/BF02091791.

Kopp, S., \& Gillberg, C. (2011). The autism Spectrum screening questionnaire (ASSQ)-revised extended version (ASSQ-REV): An instrument for better capturing the autism phenotype in girls? A preliminary study involving 191 clinical cases and community controls. Research in Developmental Disabilities, 32(6), 2875-2888. https:// doi.org/10.1016/j.ridd.2011.05.017.

Kovacs, M., \& Devlin, B. (1998). Internalizing disorders in childhood. The Journal of Child Psychology and Psychiatry and Allied Disciplines, 30(1), 47-63.

Kreiser, N. L., \& White, S. W. (2014). ASD in females: Are we overstating the gender difference in diagnosis? Clinical Child and Family Psychology Review, 17(1), 67-84. https://doi.org/10.1007/ s10567-013-0148-9.

Lai, M.-C., \& Baron-Cohen, S. B. (2015). Identifying the lost generation of adults with autism spectrum conditions. The Lancet Psychiatry, 2(11), 1013-1027. https://doi.org/10.1016/S22150366(15)00277-1.

Lai, M.-C., Lombardo, M. V., Pasco, G., Ruigrok, A. N. V., Wheelwright, S. J., Sadek, S. A., et al. (2011). A behavioral comparison of male and female adults with high functioning autism spectrum conditions. PLoS One, 6(6), e20835. https:// doi.org/10.1371/journal.pone.0020835.

Lai, M.-C., Lombardo, M. V., Auyeung, B., Chakrabarti, B., \& BaronCohen, S. (2015). Sex/gender differences and autism: Setting the scene for future research. Journal of the American Academy of Child and Adolescent Psychiatry, 54(1), 11-24. https://doi.org/10. 1016/j.jaac.2014.10.003.

Lai, M.-C., Lombardo, M. V., Ruigrok, A. N. V., Chakrabarti, B., Auyeung, B., Szatmari, P., et al. (2017). Quantifying and exploring camouflaging in men and women with autism. Autism, 21(6), 690 702. https://doi.org/10.1177/1362361316671012.

Lai, M.-C., Lombardo, M. V., Chakrabarti, B., Ruigrok, A. N., Bullmore, E. T., Suckling, J., et al. (2019). Neural self-representation in autistic women and association with 'compensatory camouflaging'. Autism, 23(5), 1210-1223. https://doi.org/10.1177/1362361318807159.

Leadbeater, B. J., Kuperminc, G. P., Blatt, S. J., \& Hertzog, C. (1999). A multivariate model of gender differences in adolescents' internalizing and externalizing problems. Developmental Psychology, 35(5), $1268-1282$.

Levy, D., Ronemus, M., Yamrom, B., Lee, Y.-h., Leotta, A., Kendall, J., et al. (2011). Rare De novo and transmitted copy-number variation in autistic Spectrum disorders. Neuron, 70(5), 886-897. https://doi. org/10.1016/j.neuron.2011.05.015.

Livingston, L. A., \& Happé, F. (2017). Conceptualising compensation in neurodevelopmental disorders: Reflections from autism Spectrum disorder. Neuroscience and Biobehavioral Reviews, 80, 729-742. https://doi.org/10.1016/j.neubiorev.2017.06.005.

Livingston, L. A., Colvert, E., Bolton, P., \& Happé, F. (2019). Good social skills despite poor theory of mind: Exploring compensation 
in autism spectrum disorder. Journal of Child Psychology and Psychiatry, 60(1), 102-110. https://doi.org/10.1111/jcpp.12886.

Loomes, R., Hull, L., \& Mandy, W. P. L. (2017). What is the male-tofemale ratio in autism Spectrum disorder? A systematic review and meta-analysis. Journal of the American Academy of Child \& Adolescent Psychiatry, 56(6), 466-474. https://doi.org/10.1016/j. jaac.2017.03.013.

Loth, E., Spooren, W., Ham, L. M., Isaac, M. B., Auriche-Benichou, C., Banaschewski, T., et al. (2015). Identification and validation of biomarkers for autism spectrum disorders. Nature Reviews Drug Discovery, 15(1), 70-73. https://doi.org/10.1038/nrd.2015.7.

Mandy, W., \& Tchanturia, K. (2015). Do women with eating disorders who have social and flexibility difficulties really have autism? A case series. Molecular Autism, 6(1), 6. https://doi.org/10.1186/ 2040-2392-6-6.

Mandy, W., Chilvers, R., Chowdhury, U., Salter, G., Seigal, A., \& Skuse, D. (2012). Sex differences in autism spectrum disorder: Evidence from a large sample of children and adolescents. Journal of Autism and Developmental Disorders, 42(7), 1304-1313. https://doi.org/ 10.1007/s10803-011-1356-0.

Mandy, W., Pellicano, L., St Pourcain, B., Skuse, D., \& Heron, J. (2018). The development of autistic social traits across childhood and adolescence in males and females. Journal of Child Psychology and Psychiatry, 59(11), 1143-1151. https://doi.org/10.1111/jcpp.12913.

Mattila, M.-L., Kielinen, M., Linna, S.-L., Jussila, K., Ebeling, H., Bloigu, R., et al. (2011). Autism Spectrum disorders according to DSM-IV-TR and comparison with DSM-5 draft criteria: An epidemiological study. Journal of the American Academy of Child \& Adolescent Psychiatry, 50(6), 583-592.e11. https://doi.org/10. 1016/j.jaac.2011.04.001.

May, T., Cornish, K., \& Rinehart, N. J. (2012). Gender profiles of behavioral attention in children with autism Spectrum disorder. Journal of Attention Disorders, 20(7), 627-635. https://doi.org/10.1177/ 1087054712455502.

McFayden, T. C., Albright, J., Muskett, A. E., \& Scarpa, A. (2018). Brief report: Sex differences in ASD diagnosis - A brief report on restricted interests and repetitive behaviors. Journal of Autism and Developmental Disorders, 49(4), 1693-1699. https://doi.org/10. 1007/s10803-018-3838-9.

Milner, V., McIntosh, H., Colvert, E., \& Happé, F. (2019). A qualitative exploration of the female experience of autism Spectrum disorder (ASD). Journal of Autism and Developmental Disorders, O(0), 114. https://doi.org/10.1007/s10803-019-03906-4.

Mpaka, D. M., Okitundu, D. L. E. A., Ndjukendi, A. O., N'situ, A. M., Kinsala, S. Y., Mukau, J. E., et al. (2016). Prevalence and comorbidities of autism among children referred to the outpatient clinics for neurodevelopmental disorders. The Pan African Medical Journal, 25, 82. https://doi.org/10.11604/pamj.2016.25.82.4151.

Murphy, C., Wilson, C. E., Robertson, D. M., Ecker, C., Daly, E. M., Hammond, N., et al. (2016). Autism spectrum disorder in adults: Diagnosis, management, and health services development. Neuropsychiatric Disease and Treatment, 12, 1669-1686. https:// doi.org/10.2147/NDT.S65455.

Nowell, S. W., Jones, D. R., \& Harrop, C. (2019). Circumscribed interests in autism: Are there sex differences? Advances in Autism, AIA-092018-0032. https://doi.org/10.1108/AIA-09-2018-0032.

Ormond, S., Brownlow, C., Garnett, M. S., Rynkiewicz, A., \& Attwood, T. (2018). Profiling autism symptomatology: An exploration of the Q-ASC parental report scale in capturing sex differences in autism. Journal of Autism and Developmental Disorders, 48(2), 389-403. https://doi.org/10.1007/s10803-017-3324-9.

Oswald, T. M., Winter-Messiers, M. A., Gibson, B., Schmidt, A. M., Herr, C. M., \& Solomon, M. (2016). Sex differences in internalizing problems during adolescence in autism Spectrum disorder. Journal of Autism and Developmental Disorders, 46(2), 624-636. https:// doi.org/10.1007/s10803-015-2608-1.
Ozonoff, S., Young, G., Carter, A., Messinger, D., Yirmiya, N., Zwaigenbaum, L., et al. (2011). Recurrence risk for autism spectrum disorders: A baby siblings research consortium study. Pediatrics, 128(3), 488-495. https://doi.org/10.1542/peds.2010-2825.

Parish-Morris, J., Liberman, M. Y., Cieri, C., Herrington, J. D., Yerys, B. E., Bateman, L., et al. (2017). Linguistic camouflage in girls with autism spectrum disorder. Molecular Autism, 8(1), 48. https://doi. org/10.1186/s13229-017-0164-6.

Randall, M., Sciberras, E., Brignell, A., Ihsen, E., Efron, D., Dissanayake, C., \& Williams, K. (2016). Autism spectrum disorder: Presentation and prevalence in a nationally representative Australian sample. Australian and New Zealand Journal of Psychiatry, 50(3), 243253. https://doi.org/10.1177/0004867415595287.

Ratto, A. B., Kenworthy, L., Yerys, B. E., Bascom, J., Trubanova, A., White, S. W., et al. (2018). What about the girls ? Sex-based differences in autistic traits and adaptive skills. Journal of Autism and Developmental Disorders, 48(5), 1698-1711. https://doi.org/10. 1007/s10803-017-3413-9.

Rivet, T. T., \& Matson, J. L. (2011). Review of gender differences in core symptomatology in autism spectrum disorders. Research in Autism Spectrum Disorders, 23(3), 957-976. https://doi.org/10.1007/ s10882-011-9235-3.

Robinson, E. B., Lichtenstein, P., Anckarsäter, H., Happé, F., \& Ronald, A. (2013). Examining and interpreting the female protective effect against autistic behavior. Proceedings of the National Academy of Sciences of the United States of America, 110(13), 5258-5262. https://doi.org/10.1073/pnas.1211070110.

Russell, G., Steer, C., \& Golding, J. (2011). Social and demographic factors that influence the diagnosis of autistic spectrum disorders. Social Psychiatry and Psychiatric Epidemiology, 46(12), 12831293. https://doi.org/10.1007/s00127-010-0294-z.

Russell, G., Rodgers, L. R., Ukoumunne, O. C., \& Ford, T. (2014). Prevalence of parent-reported ASD and ADHD in the UK: Findings from the millennium cohort study. Journal of Autism and Developmental Disorders, 44(1), 31-40. https://doi.org/10.1007/ s10803-013-1849-0.

Rutherford, M., McKenzie, K., Johnson, T., Catchpole, C., O'Hare, A., McClure, I., et al. (2016). Gender ratio in a clinical population sample, age of diagnosis and duration of assessment in children and adults with autism spectrum disorder. Autism : The International Journal of Research and Practice, 1362361315617879. https://doi. org $/ 10.1177 / 1362361315617879$.

Rynkiewicz, A., Schuller, B., Marchi, E., Piana, S., Camurri, A., Lassalle, A., \& Baron-Cohen, S. (2016). An investigation of the "female camouflage effect' in autism using a computerized ADOS-2 and a test of sex/gender differences. Molecular Autism, 7(10), 1. https://doi.org/ 10.1186/s13229-016-0073-0.

Saemundsen, E., Magnússon, P., Georgsdóttir, I., Egilsson, E., \& Rafnsson, V. (2013). Prevalence of autism spectrum disorders in an Icelandic birth cohort. BMJ Open, 3(6), 1-7. https://doi.org/10. 1136/bmjopen-2013-002748.

Schwarz, E., Guest, P. C., Rahmoune, H., Wang, L., Levin, Y., Ingudomnukul, E., et al. (2011). Sex-specific serum biomarker patterns in adults with Asperger's syndrome. Molecular Psychiatry, 16, 1213-1220. https://doi.org/10.1038/mp.2010.102.

Sedgewick, F., Hill, V., \& Pellicano, E. (2019). 'It's different for girls': Gender differences in the friendships and conflict of autistic and neurotypical adolescents. Autism, 23(5), 1119-1132. https://doi. org $/ 10.1177 / 1362361318794930$.

Shattuck, P. T., Durkin, M., Maenner, M., Newschaffer, C., Mandell, D. S., Wiggins, L., et al. (2009). Timing of identification among children with an autism spectrum disorder: Findings from a populationbased surveillance study. Journal of the American Academy of Child and Adolescent Psychiatry, 48(5), 474-483. https://doi.org/10.1097/ CHI.0b013e31819b3848. 
Skuse, D. H. (2000). Imprinting, the X-chromosome, and the male brain: Explaining sex differences in the liability to autism. Pediatric Research, 47, 9.

Strang, J. F., Powers, M. D., Knauss, M., Sibarium, E., Leibowitz, S. F., Kenworthy, L., et al. (2018). "They thought it was an obsession": Trajectories and perspectives of autistic transgender and genderdiverse adolescents. Journal of Autism and Developmental Disorders, 48(12), 4039-4055. https://doi.org/10.1007/s10803018-3723-6.

Sutherland, R., Hodge, A., Bruck, S., Costley, D., \& Klieve, H. (2017). Parent-reported differences between school-aged girls and boys on the autism spectrum. Autism, 21(6), 785-794. https://doi.org/10. $1177 / 1362361316668653$.

Tick, B., Bolton, P., Happé, F., Rutter, M., \& Rijsdijk, F. (2016). Heritability of autism spectrum disorders: A meta-analysis of twin studies. Journal of Child Psychology and Psychiatry and Allied Disciplines, 57(5), 585-595. https://doi.org/10.1111/jcpp.12499.

Tierney, S., Burns, J., \& Kilbey, E. (2016). Looking behind the mask: Social coping strategies of girls on the autistic spectrum. Research in Autism Spectrum Disorders, 23, 73-83. https://doi.org/10.1016/j. rasd.2015.11.013.

Van Wijngaarden-Cremers, P. J. M., van Eeten, E., Groen, W. B., Van Deurzen, P. A., Oosterling, I. J., \& Van der Gaag, R. J. (2014).
Gender and age differences in the core triad of impairments in autism spectrum disorders: A systematic review and meta-analysis. Journal of Autism and Developmental Disorders, 44(3), 627-635. https://doi.org/10.1007/s10803-013-1913-9.

Wilson, C. E., Murphy, C. M., McAlonan, G., Robertson, D. M., Spain, D., Hayward, H., et al. (2016). Does sex influence the diagnostic evaluation of autism spectrum disorder in adults? Autism, 20(7), 808-819. https://doi.org/10.1177/1362361315611381.

Wing, L. (1981). Sex ratios in early childhood autism and related conditions. Psychiatry Research, 5(2), 129-137. https://doi.org/10.1016/ 0165-1781(81)90043-3.

World Health Organization. (2018). The ICD-11. International Classification of Diseases for Mortality and Morbidity Statistics Eleventh Revision. https://icd.who.int/

Yeargin-Allsopp, M., Rice, C., Karapurkar, T., Doernberg, N., Boyle, C., \& Murphy, C. (2003). Prevalence of autism in a US metropolitan area. JAMA, 289(1), 49-55. https://doi.org/10.1001/jama.289.1.49.

Publisher's Note Springer Nature remains neutral with regard to jurisdictional claims in published maps and institutional affiliations. 